University of Wollongong

Research Online

Australian Institute for Innovative Materials -

Papers

Australian Institute for Innovative Materials

$1-1-2017$

\title{
High-strength graphene and polyacrylonitrile composite fiber enhanced by surface coating with polydopamine
}

Hyunsoo Kim

Hanyang University

Rouhollah Jalili

Royal Melbourne Institute of Technology, rjalili@uow.edu.au

Geoffrey M. Spinks

University of Wollongong, gspinks@uow.edu.au

Gordon G. Wallace

University of Wollongong, gwallace@uow.edu.au

Seon Jeong Kim

Hanyang University, sjk@hanyang.ac.kr

Follow this and additional works at: https://ro.uow.edu.au/aiimpapers

Part of the Engineering Commons, and the Physical Sciences and Mathematics Commons

Research Online is the open access institutional repository for the University of Wollongong. For further information contact the UOW Library: research-pubs@uow.edu.au 


\title{
High-strength graphene and polyacrylonitrile composite fiber enhanced by surface coating with polydopamine
}

\author{
Abstract \\ Carbon fibers are well-known reinforcing elements in advanced composites, but these materials remain \\ expensive partly due to the complex processing methods used to form high strength and high modulus \\ fibers. Graphene is seen as an alternative precursor for the formation of high strength carbon-based \\ fibers. Here it is shown that the strength and modulus of graphene-based fibers are enhanced by \\ incorporating a polyacrylonitrile (PAN) binder, surface coating with polydopamine (PDA) and through \\ appropriate pyrolysis heat treatments. Fiber samples were prepared by a wet-spinning method such that \\ the composition of liquid-crystalline graphene oxide (LCGO) and PAN could be varied over the full range. \\ The maximum fiber mechanical strength (220 MPa) and modulus (19 GPa) occurred at a composition of \\ LCGO (80 wt\%) and PAN (20 wt\%). The mechanical strength was further significantly increased to 526 \\ MPa through pyrolysis of the LCGO/PAN fiber at $800{ }^{\circ} \mathrm{C}$ in a nitrogen atmosphere which caused \\ carbonization of PAN. In addition, surface treatment of the LCGO/PAN fiber with PDA before \\ carbonization improved the mechanical strength by an additional $40 \%$.

\section{Disciplines} \\ Engineering | Physical Sciences and Mathematics

\section{Publication Details} \\ Kim, H., Jalili, R., Spinks, G. M., Wallace, G. G. \& Kim, S. Jeong. (2017). High-strength graphene and \\ polyacrylonitrile composite fiber enhanced by surface coating with polydopamine. Composites Science \\ and Technology, 149 280-285.
}




\section{High-strength Graphene and Polyacrylonitrile Composite Fiber Enhanced by Surface Coating with Polydopamine}

Hyunsoo Kim ${ }^{1}$, Rouhollah Jalili², Geoffrey M. Spinks ${ }^{3}$, Gordon G. Wallace ${ }^{3}$, and Seon Jeong $\mathrm{Kim}^{1, *}$

${ }^{1}$ Center for Self-Powered Actuation, Department of Biomedical Engineering, Hanyang University, Seoul 04763, Korea

${ }^{2}$ School of Science, College of Science, Engineering and Health, RMIT University, Melbourne, VIC 3001, Australia

${ }^{3}$ Intelligent Polymer Research Institute, ARC Centre of Excellence for Electromaterials Science, AIIM Facility, Innovation Campus, University of Wollongong, North Wollongong, NSW 2522, Australia

*Corresponding author: Seon Jeong Kim (sjk@hanyang.ac.kr)

\section{Abstract}

Carbon fibers are well-known reinforcing elements in advanced composites, but these materials remain expensive partly due to the complex processing methods used to form high strength and high modulus fibers. Graphene is seen as an alternative precursor for the formation of high strength carbon-based fibers. Here it is shown that the strength and modulus of graphene-based fibers are enhanced by incorporating a polyacrylonitrile (PAN) binder, surface coating with polydopamine (PDA) and through appropriate pyrolysis heat treatments. Fiber samples were prepared by a wet-spinning method such that the composition of liquid-crystalline graphene oxide (LCGO) and PAN could be varied over the full range. The maximum fiber mechanical strength (220 $\mathrm{MPa})$ and modulus (19 GPa) occurred at a composition of LCGO (80 wt $\%$ ) and PAN (20 wt\%). The mechanical strength was further significantly increased to $526 \mathrm{MPa}$ 
through pyrolysis of the LCGO/PAN fiber at $800{ }^{\circ} \mathrm{C}$ in a nitrogen atmosphere which caused carbonization of PAN. In addition, surface treatment of the LCGO/PAN fiber with PDA before carbonization improved the mechanical strength by an additional $40 \%$.

Keywords: Graphene oxide, polyacrylonitrile, surface coating, pyrolysis, high strength.

\section{Introduction}

Carbon based fibers show high specific strengths and specific modulus and have found increasing application for production of high performance carbon composite components, bulletproof vests and other protective garments [1-3]. Polyacrylonitrile (PAN) and its copolymers are the most common precursors for the production of carbon fibers but very high molecular weight with very low defects are required. Moreover, a series of thermal treatments must be carried out to convert the PAN fiber to high tenacity carbon fiber, including thermal stabilization $\left(\sim 300{ }^{\circ} \mathrm{C}\right)[4]$, carbonization $\left(\sim 1000^{\circ} \mathrm{C}\right)$ and graphitization $\left(>2000{ }^{\circ} \mathrm{C}\right)$ [5]. Difficulties in the synthesis of the PAN precursors and the elevated temperatures needed for pyrolysis motivate research into more cost-effective and simpler routes to produce carbon-based fibers.

Recently, carbon nanotube (CNT) and graphene fibers prepared using simple wet-spinning techniques have been developed as high-strength and high-stiffness fibers. The mechanical strength and modulus of wet-spun CNT fibers have been reported at 1.3 $\mathrm{GPa}$ and $150 \mathrm{GPa}$ [6], respectively. Zhang et al. reported a tensile strength of $850 \mathrm{MPa}$ when the CNT fiber was infiltrated with polyvinyl alcohol. In this case the CNT fiber was fabricated by dry spinning from a multi-walled CNT forest [7]. Although CNT fibers show high mechanical performance, wet spinning is limited by dispersion 
stability, and the dry-spinning technique is costly to up-scale [8]. The graphene precursor liquid crystalline graphene oxide (LCGO) can be readily and easily processed into a wide variety of structures including fibers [9-13]. High performance graphene fibers with tensile strength and modulus of $442 \mathrm{MPa}$ and $22.6 \mathrm{GPa}$, respectively, have been produced using a one-step wet-spinning method [9]. Also, various composite graphene fibers have been prepared using different polymer binders. The reported tensile strengths were in the range of $150-450 \mathrm{MPa}$ for PAN binder $[14,15] ; \sim 360$ MPa for cellulose binder [16]; and $\sim 178 \mathrm{MPa}$ for a polyetherimide/graphene composite where polydopamine (PDA) was grafted to graphene oxide [17]. Although improvements have been made in the mechanical properties of graphene fiber, the results are still short of commercially available carbon fibers.

Surface treatment has proven to be a reliable method to modify the mechanical performance of fibers [18]. Gao et al. [18] showed enhanced mechanical strength of glass fiber by applying a CNT coating on the fiber surface. Such coatings can reduce the incidence of surface defects that reduce fiber strength. Indeed, Ryu et al. [19] dramatically improved the tensile strength of CNT yarn from $1004 \mathrm{MPa}$ to $4035 \mathrm{MPa}$ by applying a PDA coating on the CNT surface and through a pyrolysis heat treatment. PDA is known to spontaneously adhere to the surface of various solid materials [20], and has been used to form coatings for various applications such as hydrophilic and biocompatible properties [21], and core/shell nanostructure [22].

The effects of composite composition, PDA surface treatments and pyrolysis on the mechanical properties of PAN/graphene fibers are reported here. The composite composition was systematically varied to determine the optimum level of graphene reinforcement and then the PDA surface coating / pyrolysis methods were applied to 
investigate strength and stiffness enhancement mechanisms.

\section{Experimental methods}

\subsection{Materials}

Dispersed liquid crystalline graphene oxide (LCGO) $(10 \mathrm{mg} / \mathrm{ml})$ in water was prepared using previously published methods [9, 11, 23], the mean size of GO flakes was $37 \pm 23 \mu \mathrm{m}$. PAN homopolymer (molecular weight: 200,000) was purchased from Polysciences, Inc. (USA). Dopamine hydrochloride was purchased from Sigma Aldrich (USA).

\subsection{Fabrication and pyrolysis of LCGO/PAN/PDA fiber}

To prepare composite PAN and LCGO fibers by wet spinning, the as-received aqueous dispersions of LCGO were first converted to a N,N-dimethylformamide (DMF) dispersion. The LCGO was collected by centrifugation of the water dispersion and supernatant water was removed. Next, the graphene was re-dispersed in solvent mixture of equal volumes of water and DMF. The solid was again recovered by centrifugation and the same process was repeated a minimum of six times to remove water. Finally, the collect LCGO flakes were dispersed in DMF (10 wt $\%)$ and mixed with a PAN/DMF solution (10 wt $\%)$ using a vortex mixer. The proportions of LCGO/DMF and PAN/DMF were varied to control the final composite composition. LCGO/PAN fibers were fabricated by wet spinning by extruding the spinning dispersion through a 22-guage spinneret into a coagulation bath of mixed water/isopropyl alcohol (IPA) (7:3) solution. When preparing the neat LCGO fiber an addition of $1 \mathrm{wt} \%$ calcium chloride was added to the coagulation bath. The LCGO/PAN solution injection speed was $5-20 \mathrm{ml} / \mathrm{h}$ and 
the coagulation bath was rotation at $20-30 \mathrm{rpm}$ to form continuous fibers. After spinning, LCGO/PAN fiber was removed from the coagulation bath and completely dried in air atmosphere at $280^{\circ} \mathrm{C}$ for $1 \mathrm{~h}$. The PDA coating was applied by immersing the fiber in aqueous dopamine (Tris- $\mathrm{HCl}$ buffer, $\mathrm{pH}$ 8.5) solution and heating overnight at $60{ }^{\circ} \mathrm{C}$. Polymerization of the dopamine was evident from a change in color of the dopamine solution to brown. Finally, LCGO/PAN/PDA fiber underwent sufficient washing by water to remove non-polymerized DA and pyrolysis was performed at $800{ }^{\circ} \mathrm{C}$ in a nitrogen atmosphere.

\subsection{Characterization}

The thermal stability of the fiber samples was measured using thermogravimetric analysis (TGA, SDT Q600). The temperature was gradually increased from room temperature to $1000{ }^{\circ} \mathrm{C}$ at the rate of $5{ }^{\circ} \mathrm{C} / \mathrm{min}$ in air or in a nitrogen atmosphere. The ratio of the Raman intensity $\left(\mathrm{I}_{\mathrm{D}} / \mathrm{I}_{\mathrm{G}}\right)$ of the $\mathrm{D}$ and $\mathrm{G}$ peaks was obtained with a dispersive Raman spectrometer (Senterra Raman) using a $633 \mathrm{~nm}$ excitation. The mechanical properties were measured using a universal testing machine (Instron 5966) at a loading rate of $1 \mathrm{~mm} / \mathrm{min}$. The liquid crystal characteristics of the LCGO/PAN dispersions were observed using a $90^{\circ}$ polarized transmission optical microscope.

\section{Results and Discussion}

Large sheets of GO that can form liquid crystalline dispersions are a robust template for matrix-guided molecular level self-assembly of polymers [12, 23, 24]. This process allows for the production of composite materials with enhanced mechanical performance by simple mixing a polymer with LCGO $[25,26]$. Inspired by this idea, 
LCGO/PAN based composite formulations were produced in a wide range of ratios by combining the LCGO/DMF and PAN/DMF dispersions and subsequent wet-spinning to form composite fibers of controlled composition. The birefringence property of the LCGO/PAN spinning solution is shown in Fig. S1 confirming their liquid crystal character.

Fig. 1 shows the fabrication process of high tenacity graphene based PAN composite fiber by the wet-spinning method. A typical wet-spinning experiment involves injecting of the LCGO/PAN composite formulation into a rotating coagulation bath to produce the gel-state LCGO/PAN fiber (Fig. 1a). Controlling the injection speed and rotation of the coagulation bath ensures a uniform diameter in the formed gel-state LCGO/PAN fibers. Fiber stretching also occurs during fiber formation and the molecular orientation likely enhances the final fiber mechanical properties. The gel state LCGO/PAN fibers were carefully removed from the coagulation bath and then fixed and dried at room temperature, followed by heating at $280{ }^{\circ} \mathrm{C}$ for thermal stabilization in an oxygen rich atmosphere (Fig. 1b). These thermally stabilized fibers were next dipped into dopamine aqueous solution of tris buffer $(\mathrm{pH} 8.5)$ and heated at $60{ }^{\circ} \mathrm{C}$. The dopamine adsorbs to the exterior of the LCGO/PAN fiber and self-polymerizes on the surface [27] (Fig. 1c). Finally, the pyrolysis of the fibers was carried out at $800{ }^{\circ} \mathrm{C}$, in nitrogen, to carbonize the PAN - PDA fibers. The pyrolysis process results in the carbonization of the composite fibers (Fig. 1d).

The tensile strength and modulus of the composite fibers as a function of PAN concentration are shown in Fig 2a, and stress-strain curves are represented in Fig S2. As the concentration of LCGO in the composite fibers was increased, the tensile strength also increased until a peak of $220.1 \pm 28.2 \mathrm{MPa}$ was achieved at $80 \mathrm{wt} \%$ LCGO. 
Samples prepared at $90 \mathrm{wt} \%$ and $100 \mathrm{wt} \%$ LCGO showed lower tensile strengths. The elastic modulus of the fibers showed a similar trend as it increased by increasing the LCGO concentration of up to $90 \mathrm{wt} \%$ and then decreased for the neat $100 \mathrm{wt} \%$ LCGO fiber. The measured densities of the $100 \mathrm{wt} \%$ LCGO fiber $\left(1.1 \mathrm{~g} / \mathrm{cm}^{3}\right)$ and $80 \mathrm{wt} \%$ LCGO fiber $\left(1.51 \mathrm{~g} / \mathrm{cm}^{3}\right)$ suggested that the PAN polymer occupied much of the vacant space between the large GO sheets. The reduction in void space with PAN increases the modulus, as expected from the rule-of-mixtures model where the composite modulus is a weighted average of the moduli of the two components. The increase in modulus also leads to an increase in tensile strength since the strain at failure remains relatively unchanged for all composites and the pure LCGO fibers [28]. In contrast, the strain at failure of the neat PAN fiber was considerably higher than the composites. The presence of stress concentrations, such as void space or imperfections at the LCGO flake/PAN interface, will induce brittleness and reduce the strain at failure. At high LCGO loadings, the amount of PAN binder becomes insufficient to completely fill the void space and strength and modulus are then reduced.

PDA coating and pyrolysis were performed on the LCGO/PAN fiber containing $80 \mathrm{wt} \%$ of LCGO. An ordered inner structure of aligned LCGO flakes was observed in the as-spun gel-state LCGO/PAN fiber using the polarized optical microscopy, (Fig. 2b). Drying reduced the diameter of the LCGO/PAN fiber by a factor of 10 from $248 \pm 0.2$ $\mu \mathrm{m}$ to $25 \pm 0.1 \mu \mathrm{m}$. The large volume decrease also generated a wrinkled surface (Fig. $2 \mathrm{~b}$, ii) on the LCGO/PAN fiber. The cross section of the LCGO/PAN fiber with $80 \mathrm{wt} \%$ LCGO had a compact structure, as shown in Fig. 2c. Topographical features on the fiber cross-section are consistent with stacked GO flakes aligned along the fiber axis [9].

The effects of processing conditions on the mechanical properties of the 
LCGO/PAN fibers were investigated. Fig. S3 show the stress-strain curves for fibers prepared by controlling the speed of the solution injection, and the rotating speed of the coagulation bath. Varying the solution pumping rate from 5 to $20 \mathrm{ml} / \mathrm{h}$ at the same bath rotation speed $(20 \mathrm{rpm})$ had only a small effect on the tensile strength $(150-175 \mathrm{MPa})$ and modulus (15 -16 GPa). However, increasing rotation speed of the coagulation bath to $30 \mathrm{rpm}$, with a pumping rate of $5 \mathrm{ml} / \mathrm{h}$, generated a significant increase in the tensile strength $(220.1 \mathrm{MPa})$ and modulus $(19.7 \mathrm{GPa})$. The combination of the slow pumping rate and high coagulation bath rotation speed induced the highest stretch and the best mechanical performance, suggesting better alignment of the GO flakes.

The thermal behavior of the LCGO/PAN/PDA fiber was investigated by TGA. Fig. 3a (inset) shows the TGA curve of the LCGO/PAN fiber in air. The LCGO/PAN fiber showed significant weight decrease of $\sim 25 \%$ between $180{ }^{\circ} \mathrm{C}$ and $300{ }^{\circ} \mathrm{C}$. As reported previously, thermal treatment of PAN between $200{ }^{\circ} \mathrm{C}$ and $300{ }^{\circ} \mathrm{C}$ in an oxygen rich condition causes the nitrile groups to form an aromatic ladder structure $[4,5]$. This aromatization facilitates the further annealing of PAN at high temperature to increase the yield of carbonization [5]. The weight loss during the pyrolysis stage of heat treatment is also shown in Fig. 3a for the LCGO/PAN fiber coated with PDA. Heating to $800{ }^{\circ} \mathrm{C}$ in nitrogen causes a weight loss of $\sim 20 \%$ because of the carbonization process. A similar weight loss during pyrolysis was observed for the LCGO/PAN fibers without the PDA coating. The Raman spectrum in Fig. 3b shows typical D band and G bands, which indicate $\mathrm{sp}^{3}$ and $\mathrm{sp}^{2}$ bonds at 1350 and $1580 \mathrm{~cm}^{-1}$, respectively. The intensity ratio of $D$ peak and $G$ peaks $\left(\mathrm{I}_{\mathrm{D}} / \mathrm{I}_{\mathrm{G}}\right)$ gives an estimate of the amount of defects in the graphene structure [27]. The pyrolysed LCGO/PAN/PDA had a smaller peak intensity ratio of 1.3 in comparison to the non-pyrolysed LCGO/PAN/PDA (1.6). The smaller 
intensity ratio of $\mathrm{I}_{\mathrm{D}} / \mathrm{I}_{\mathrm{G}}$ indicates a conversion of $\mathrm{sp}^{3}$ to $\mathrm{sp}^{2}$ bonds due to pyrolysis. Carbonization is known to remove oxygen-containing functional groups, such as epoxy and carboxyl groups, from the surface of the LCGO and hydrogen bonding with PAN [29]. In addition, the diameter of the fibers decreased from $\sim 25 \pm 0.1 \mu \mathrm{m}$ (Fig. 2b) to $\sim 19 \pm 0.1 \mu \mathrm{m}$ (Fig. 4a, b) during pyrolysis giving a more compact structure in the pyrolysed LCGO/PAN/PDA fiber (Fig. 3c) in comparison with the LCGO/PAN fiber (Fig. 2c). Fig. 4c, d shows magnified images of surface morphologies of the pyLCGO/PAN, and py-LCGO/PAN/PDA fiber. The py-LCGO/PAN has a wrinkled structure, while a smoother surface was observed on the py-LCGO/PAN/PDA.

The mechanical strength and elastic moduli of the fibers were affected by four factors. Firstly, the well-aligned inner structure of the large size GO sheets produces effective mechanical reinforcement in comparison with smaller size of GO. Secondly, as the PAN matrix binder filled many of the microvoids in the LCGO fiber, the mechanical strength and stiffness was improved. Density is further increased through the pyrolysis, so the py-LCGO/PAN fiber is more compact by carbonization of PAN and reducing GO. The reduction in oxide defects allows for a more compact structure and resulting in the tensile strength and modulus of LCGO/PAN fiber increasing from $220.1 \pm 28.2 \mathrm{MPa}$ to $526.2 \pm 28.7 \mathrm{MPa}$ and from $19 \pm 1.9 \mathrm{GPa}$ to $72.6 \pm 10.1 \mathrm{GPa}$, respectively (Fig. 5). Finally, the PDA coating induced higher tensile strength as PDA covered the surface defects of the LCGO/PAN fiber. The surface defects act as stress concentrations and induce fracture by cracking at low stress [18]. Hence, the tensile strength of pyLCGO/PAN increased $40 \%$ and reached $738.5 \pm 25.3 \mathrm{MPa}$. The modulus of $73.2 \pm 13.7$ GPa obtained for PDA coated fibers was unchanged from the uncoated fibers. The LCGO/PAN fiber enhanced by PDA coating has a higher tensile strength than most 
previously reported graphene oxide based materials (Table 1). Composite fiber and films formed from graphene oxide have been reported with tensile strengths in the range $148 \mathrm{MPa}$ to $652 \mathrm{MPa}[16,17,30-32]$ The tensile strength and modulus of the pyLCGO/PAN/PDA fiber is also comparable to thermally annealed at $800-1050{ }^{\circ} \mathrm{C} \mathrm{GO}$ fibers [33-35], and the graphene based fibers [36-41].

\section{Conclusion}

In conclusion, we demonstrated an enhancement in the strength and modulus of LCGO/PAN composite fiber through surface coating with PDA and pyrolysis. The fibers were prepared from a simple wet spinning method using mixed dispersions of LCGO and PAN to control the final fiber composition. The highest tensile strength for non-coated and non-pyrolysed LCGO/PAN fibers (220.1 MPa) was observed for $80 \mathrm{wt} \%$ of LCGO and $20 \mathrm{wt} \%$ of PAN fiber due to the large aligned surface of GO and high GO loading. In addition, after the surface treatment of PDA and pyrolysis, a dramatic improvement of mechanical strength and modulus was observed, $738.5 \pm 25.3 \mathrm{MPa}$ and $73.2 \pm 13.7 \mathrm{GPa}$, respectively. As a result, the high mechanical strength of pyLCGO/PAN/PDA fiber has the potential to act as a reinforcing agent for increasing mechanical properties of composite materials.

\section{Acknowledgment}

This work was supported by the Creative Research Initiative Center for Self-Powered Actuation in Korea. Funding was also provided from Australian Research Council (ARC) Centre of Excellence Scheme (CE 140100012). The 
authors gratefully acknowledge the materials node of the Australian National Fabrication Facility (ANFF) for their facility and research support.

\section{Reference}

[1] J. Ren, W. Bai, G. Guan, Y. Zhang, H. Peng, Flexible and Weaveable Capacitor Wire Based on a Carbon Nanocomposite Fiber, Adv. Mater. 25 (2013) 5965-5970.

[2] M.K. Shin, B. Lee, S.H. Kim, J.A. Lee, G.M. Spinks, S. Gambhir, G.G. Wallace, M.E. Kozlov, R.H. Baughman, S.J. Kim, Synergistic toughening of composite fibres by self-alignment of reduced graphene oxide and carbon nanotubes, Nature commun. 3 (2012) 650.

[3] X. Zhang, Q. Li, T.G. Holesinger, P.N. Arendt, J. Huang, P.D. Kirven, T.G. Clapp, R.F. DePaula, X. Liao, Y. Zhao, L. Zheng, D.E. Peterson, Y. Zhu, Ultrastrong, Stiff, and Lightweight Carbon-Nanotube Fibers, Adv. Mater. 19 (2007) 4198-4201.

[4] D. Esrafilzadeh, M. Morshed, H. Tavanai, An investigation on the stabilization of special polyacrylonitrile nanofibers as carbon or activated carbon nanofiber precursor, Synthetic Metals 159 (2009) 267-272.

[5] M.S.A. Rahaman, A.F. Ismail, A. Mustafa, A review of heat treatment on polyacrylonitrile fiber, Polymer Degradation and Stability 92 (2007) 1421-1432. 
[6] N. Behabtu, C.C. Young, D.E. Tsentalovich, O. Kleinerman, X. Wang, A.W.K. Ma, E.A. Bengio, R.F. Waarbeek, J.J. Jong, R.E. Hoogerwerf, S.B. Fairchild, J.B. Ferguson, B. Maruyama, J. Kono, Y. Talmon, Y. Cohen, M.J. Otto, M. Pasquali, Strong, Light, Multifunctional Fibers of Carbon Nanotubes with Ultrahigh Conductivity, Science 339 (2013) 182-186.

[7] M. Zhang, K.R. Atkinson, R.H. Baughman, Multifunctional Carbon Nanotube Yarns by Downsizing an Ancient Technology, Science 306 (2004) 1358-1361.

[8] W. Lu, M. Zu, J.-H. Byun, B.-S. Kim, T.-W. Chou, State of the Art of Carbon Nanotube Fibers: Opportunities and Challenges, Adv. Mater. 24 (2012) 1805-1833.

[9] R. Jalili, S.H. Aboutalebi, D. Esrafilzadeh, R.L. Shepherd, J. Chen, S.A.Yamini, K. Konstantinov, A.I. Minett, J.M. Razal, G.G. Wallace, Scale One-Step Wet-Spinning of Graphene Fibers and Yarns from Liquid crystalline Dispersions of Graphene Oxide: Towards Multifunctional Textiles, Adv. Funct. Mater. 23 (2013) 5345-5354.

[10] S.H. Aboutalebi, R. Jalili, D. Esrafilzadeh, M. Salari, Z. Gholamvand, S.A. Yamini, K. Konstantinov, R.L. Shepherd, J. Chen, S.E. Moulton, P.C. Innis, A.I. Minett, J.M. Razal, G.G. Wallace, High-Performance Multifunctional Graphene Yarns: Toward Wearable All-Carbon Energy Storage Textiles, ACS Nano 8 (2014) 2456-2466.

[11] R. Jalili, S.H. Aboutalebi, D. Esrafilzadeh, K. Konstantinov, J.M. Razal, S.E. Moulton, G.G. Wallace, Formation and processability of liquid crystalline dispersions of graphene oxide, Mater. Horiz. 1 (2014) 87-91. 
[12] R. Jalili, S.H. Aboutalebi, D.Esrafilzadeh, K. Konstantinov, S.E. Moulton, J.M. Razal, G.G. Wallace, Organic Solvent-Based Graphene Oxide Liquid Crystals: A Facile Route toward the Next Generation of Self-Assembled Layerby-Layer Multifunctional 3D Architectures, ACS Nano 7 (2013) 3981-3990.

[13] S. Naficy, R. Jalili, S.H. Aboutalebi, R.A. Gorkin III, K. Konstantinov, P.C. Innis, G.M. Spinks, P. Poulin, G.G. Wallace, Graphene oxide dispersion: tuning rheology to enable fabrication, Mater. Horiz. 1 (2014) 326- 331.

[14] M.E. Uddin, R.K. Layek, N.H. Kim, D. Hui, J.H. Lee, Preparation and properties of reduced graphene oxide/polyacrylonitrile nanocomposites using polyvinyl phenol, Composites Part B 80 (2015) 238-245.

[15] Z. Liu, Z. Xu, X. Hu, C. Gao, Lyotropic Liquid Crystal of PolyacrylonitrileGrafted Graphene Oxide and Its Assembled Continuous Strong Nacre-Mimetic Fibers, Macromolecules 46 (2013) 6931-6941.

[16] M. Tian, L. Qu, X. Zhang, K. Zhang, S. Zhu, X. Guo, G. Han, X. Tang, Y. Sun, Enhanced mechanical and thermal properties of regenerated cellulose/graphene composite fibers, Carbohydrate Polymers 111 (2014) 456-462.

[17] Y. Tian, Y. Cao, Y. Wang, W. Yang, J. Feng, Realizing Ultrahigh Modulus and high strength of Macroscopic Graphene Oxide Papers Through Crosslinking of Mussel-Inspired Polymers, Adv. Mater. 25 (2013) 2980-2983.

[18] S.L. Gao, E. Mäder, R. Plonka, Nanostructured coating of glass fibers: Improvement of alkali resistance and mechanical properties, Acta materialia 55 (2007) 1043-1052. 
[19] S. Ryu, J. B. Chou, K. Lee, D. Lee, S.H. Hong, R. Zhao, H. Lee, S. Kim, Direct Insulation-to-Conduction Transformation of adhesive Catecholamine for Simultaneous Increases of Electrical Conductivity and Mechanical Strength of CNT Fibers, Adv. Mater. 27 (2015) 3250-3255.

[20] H. Lee, S.M. Dellatore, W.M. Miller, P.B. Messersmith, Mussel-Inspired Surface Chemistry for Multifunctional Coatings, Science 318 (2007) 426-430.

[21] X. Liu, J. Cao, H. Li, J. Li, Q. Jin, K. Ren, J. Ji, Mussel-Inspired Polydopamine: A Biocompatible and Ultrastable Coating for Nanoparticles in Vivo, ACS Nano 7 (2013) 9384-9395.

[22] L. Zhang, S. Yuan, S. Chen, D. Wang, B.-Z. Han, Z.-M. Dang, Preparation and dielectric properties of core-shell structured Ag@polydopamine/poly(vinylidene fluoride) composites, Composites science and Technology 110 (2015) 126-131.

[23] K.S.U. Schirmer, D. Esrafilzadeh, B.C. Thompson, A.F. Quigley, R.M.I. Kapsa, G.G. Wallace, Conductive composite fibres from reduced graphene oxide and polypyrrole nanoparticles, J. Mater. Chem. B 4 (2016) 1142-1149.

[24] M.M. Islam, S.H. Aboutalebi, D. Cardillo, H.K. Liu, K. Konstantinov, S.X. Dou, Self-Assembled Multifunctional Hybrids: Toward Developing HighPerformance Graphene-Based Architectures for Energy Storage Devices, ACS Cent. Sci 1 (2015) 206-216.

[25] S. Seyedin, J.M. Razal, P.C. Innis, R. Jalili, G.G. Wallace, Compositional Effects of Large Graphene Oxide Sheets on the Spinnability and Properties of Polyurethane Composite Fibers, Adv. Mater. Interfaces 3 (2016) 1500672. 
[26] M.Z. Seyedin, J.M. Razal, P.C. Innis, R. Jalili, G.G. Wallace, Achieving Outstanding Mechanical Performance in Reinforced Elastomeric Composite Fibers Using Large Sheets of Graphene Oxide, Adv. Funct. Mater. 25 (2015) 94104.

[27] K. Qu, Y. Zheng, S. Dai, S.Z. Qiao, Polydopamine-graphene oxide derived mesoporous carbon nanosheets for enhanced oxygen reduction, Nanoscale 7 (2015) 12598-12605.

[28] G. Xin, T. Yao, H. Sun, S.M. Scott, D. Shao, G. Wang, J. Lian, Highly thermally conductive and mechanically strong graphene fibers, Science 349 (2015) 1083-1087.

[29] Y. Huang, F. Lai, L. Zhang, H. Lu, Y.-E. Miao, T. Liu, Elastic Carbon Aerogels Reconstructed from Electrospun Nanofibers and Graphene as ThreeDimensional Networked Matrix for Efficient Energy Storage/Conversion, Sci. Rep. 6 (2016) 31541.

[30] J.J. Ge, H. Hou, Q. Li, M.J. Graham, A. Greiner, D.H. Reneker, F.W. Harris, S.Z.D. Cheng, Assembly of Well-Aligned Multiwalled Carbon Nanotubes in Confined Polyacrylonitrile Environments: Electrospun Composite Nanofiber Sheets, J. Am. Chem. Soc. 126 (2004) 15754-15761.

[31] X. Hu, Z. Xu, Z. Liu, C. Gao, Liquid crystal self-templating approach to ultrastrong and tough biomimic composites, Sci. Rep. 3 (2013) 2374.

[32] Y. Li, H. Zhu, S. Zhu, J. Wan, Z. Liu, O. Vaaland, S. Lacey, Z. Fang, H. Dai, T. Li, L. Hu, Hybridizing wood cellulose and graphene oxide toward highperformance fibers, NPG Asia Materials 7 (2015) 1-10. 
[33] Z. Dong, C. Jiang, H. Cheng, Y. Zhao, G. Shi, L. Jiang, L. Qu, Facile Fabrication of Light, Flexible and Multifunctional Graphene Fibers, Adv. Mater. 24 (2012) 1856-1861.

[34] C. Xiang, C.C. Young, X. Wang, Z. Yan, C.-C, Hwang, G. Cerioti, J. Lin, J. Kono, M. Pasquali, J.M. Tour, Large Flake Graphene Oxide Fibers with Unconventional $100 \%$ Knot Efficiency and Highly Aligned Small Flake Graphene Oxide Fibers, Adv. Mater. 25 (2013) 4592-4597.

[35] C. Xiang, N. Behabtu, Y. Liu, H.G. Chae, C.C. Young, B. Genorio, D.E. Tsentalovich, C. Zhang, D.V. Kosynkin, J.R. Lomeda, C.-C. Hwang, S. Kumar, M. Pasquali, J.M. Tour, Graphene Nanoribbons as an Advanced Precursor for Making Carbon Fiber, ACS Nano 7 (2013) 1628-1637.

[36] X. Zhao, Z. Xu, B. Zheng, C. Gao, Macroscopic assembled, ultrastrong and H2SO4-resistant fibres of polymer-grafted graphene oxide, Sci. Rep. 3 (2013) 3164.

[37] Z. Xu, Z. Liu, H. Sun, C. Gao, Highly Electrically, Conductive Ag-Doped Graphene Fibers as Stretchable Conductors, Adv. Mater. 25 (2013) 3249-3253.

[38] L. Chen, Y. He, S. Chai, H. Qiang, F. Chen, Qiang. Fu, Toward high performance graphene fibers, Nanoscale 5 (2013) 5809-5815.

[39] L. Kou, C. Gao, Bioinspired design and macroscopic assembly of poly(vinyl alcohol)-coated graphene into kilometers-long fibers, Nanoscale 5 (2013) 43704378.

[40] R.C.-Silva, A.M.-Gomez, H. Kim, H. Jang, F. Tristan, S.V.-Diaz, L.P. Rajukumar, A.L. Elías, N.P.-Lopez, J. Suhr, M. Endo, M. Terrones, Super- 
stretchable Graphene Oxide Macroscopic Fibers with Outstanding Knotability Fabricated by Dry Film Scrolling, ACS Nano 8 (2014) 5959-5967.

[41] S. Seyedin, M.S. Romano, A.I. Minett, J.M. Razal, Towards the Knittability of Graphene Oxide Fibres, Sci. Rep. 5 (2015) 14946. 


\section{Figure and captions}
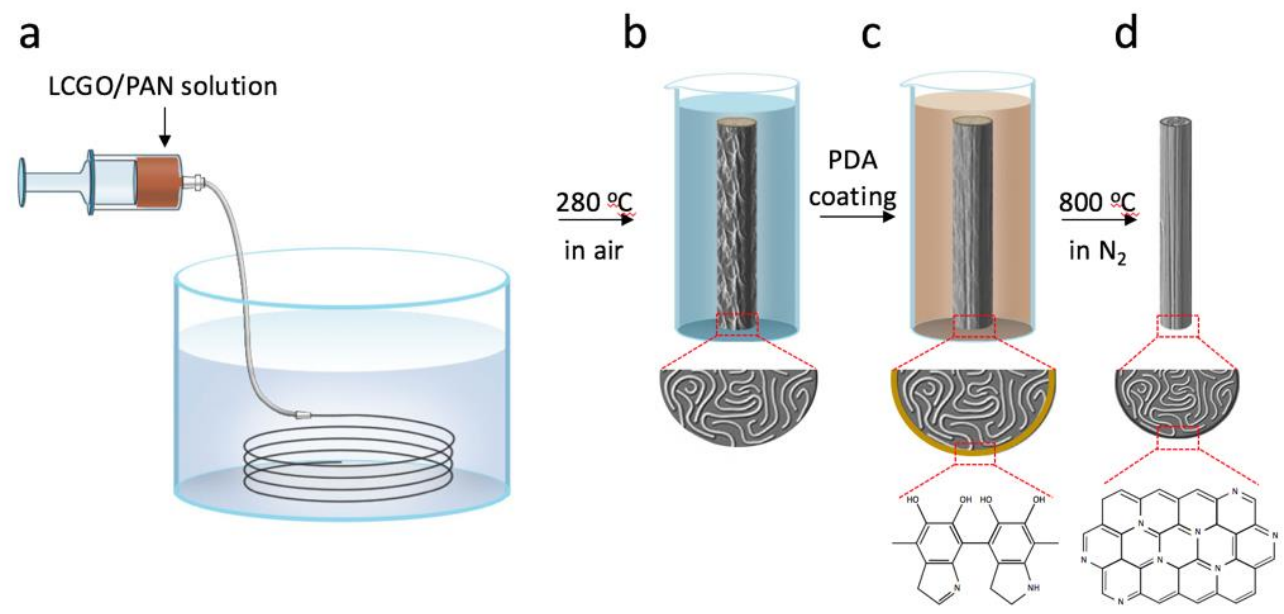

Fig. 1. Schematic diagrams illustrating a) wet spinning process of the LCGO/PAN fiber, b) thermally stabilized LCGO/PAN fiber immersed in DA solution, c) the polydopamine (PDA) self-polymerization on the surface of the LCGO/PAN fiber, and d) pyrolysis of LCGO/PAN/PDA fiber at $800{ }^{\circ} \mathrm{C}$ in $\mathrm{N}_{2}$ atmosphere. 

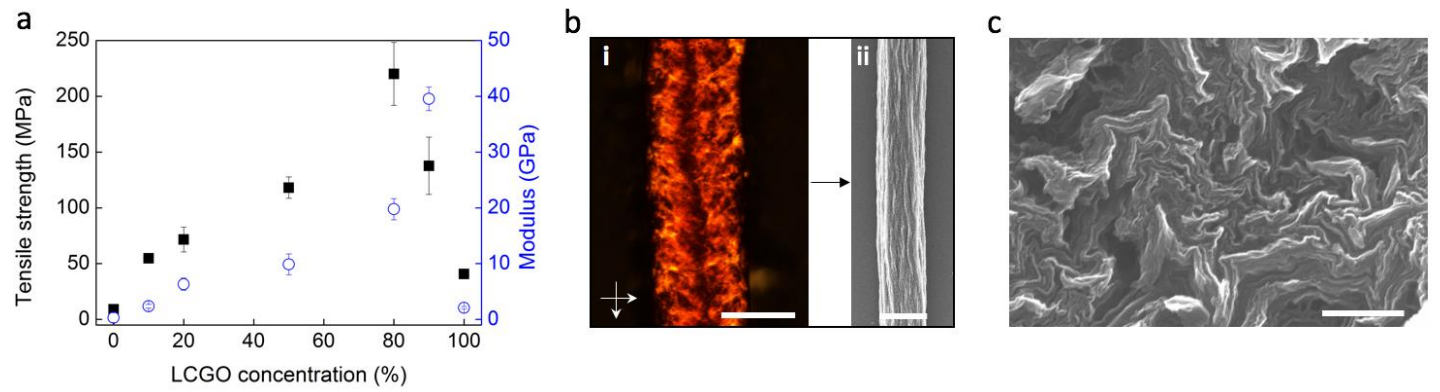

Fig. 2. a) Tensile strength and modulus of the composite fiber versus LCGO concentration. b) i) Polarized optical microscopy image of gel-state LCGO/PAN fiber showing LC domains (arrows show the polarizer directions) (scale bar: $200 \mu \mathrm{m}$ ). ii) SEM image of the surface of dried LCGO/PAN fiber (scale bar: $25 \mu \mathrm{m}$ ). c) SEM image of the cross section of the LCGO/PAN fiber (scale bar: $500 \mathrm{~nm})$. 

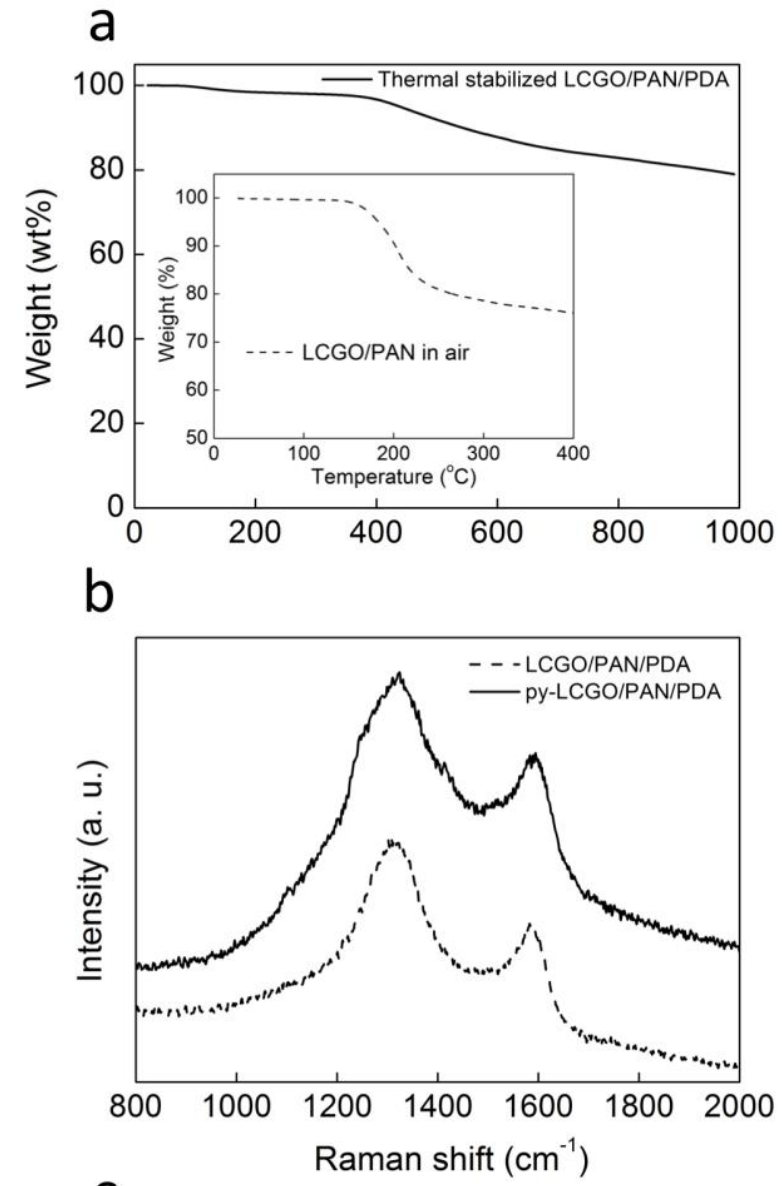

C

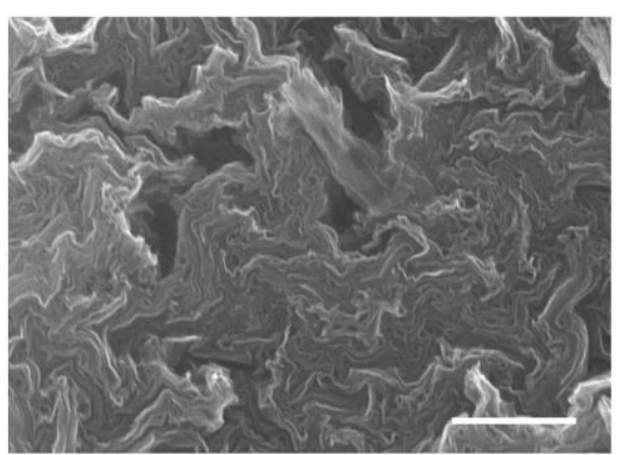

Fig. 3. a) TGA curves of the thermally stabilized LCGO/PAN/PDA fiber in $\mathrm{N}_{2}$ atmosphere. Inset is TGA curves of the LCGO/PAN fiber in air condition. b) Raman spectroscopy of the LCGO/PAN/PDA (dashed line), and pyLCGO/PAN/PDA (solid line). c) Cross section of py-LCGO/PAN/PDA (Scale bar: $500 \mathrm{~nm})$. 

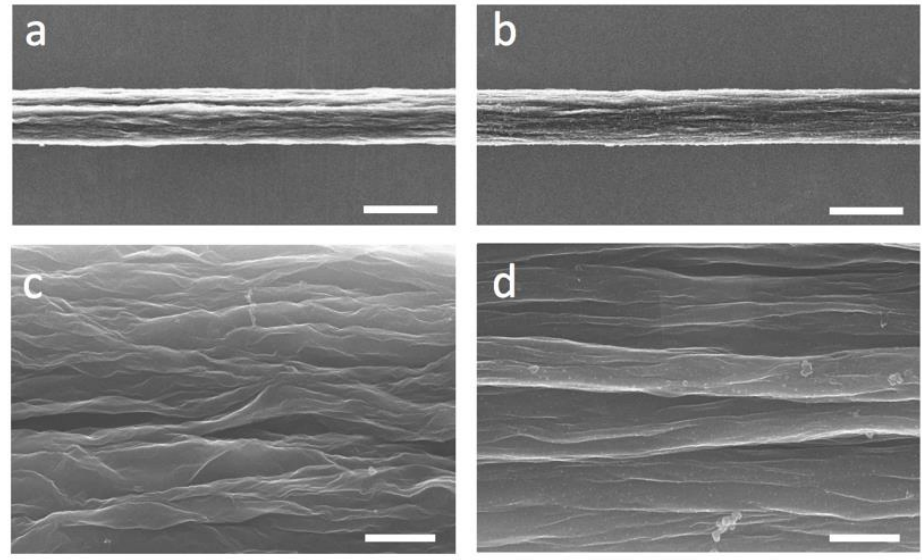

Fig. 4. SEM images of the surface of a) py-LCGO/PAN, and b) pyLCGO/PAN/PDA fiber (scale bar: $25 \mu \mathrm{m}$ ). Magnified surface of c) pyLCGO/PAN, and d) py-LCGO/PAN/PDA fiber (scale bar: $1 \mu \mathrm{m}$ ). 


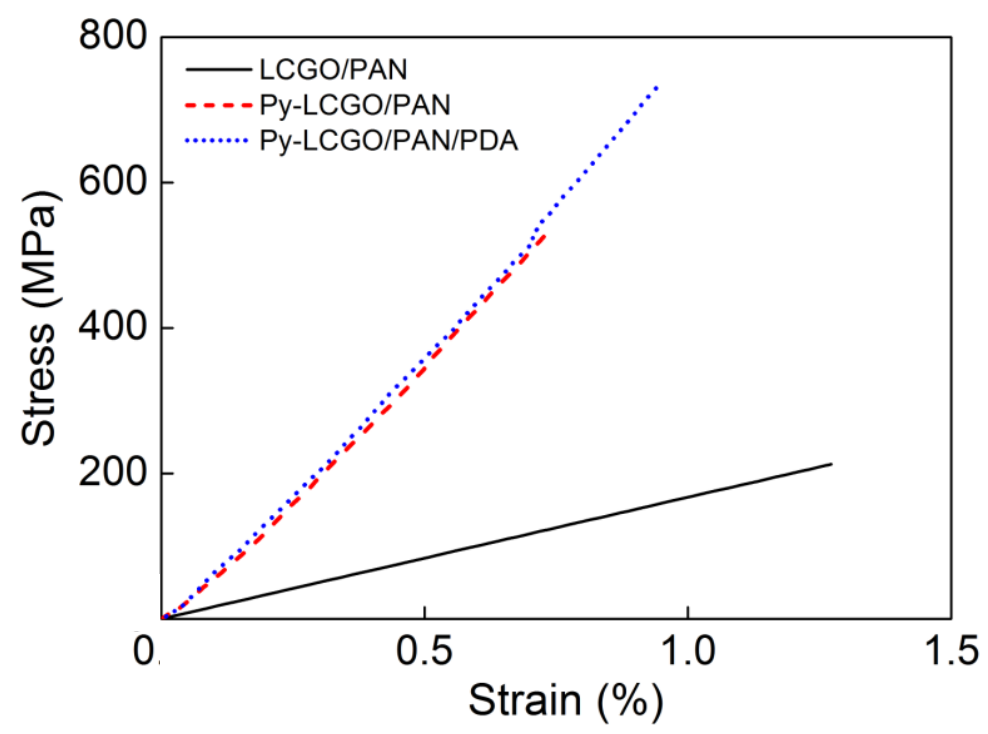

Fig. 5. Stress-strain curves of the LCGO/PAN fiber (solid line), py-LCGO/PAN (dashed line), and py-LCGO/PAN/PDA (dotted line). 
Table. 1. Tensile strength and Young's modulus of GO based fibers prepared in this study and previous reports.

\begin{tabular}{ccccc}
\hline Reference & Species & Method & $\begin{array}{c}\text { Tensile strength } \\
{[\mathrm{MPa}]}\end{array}$ & $\begin{array}{c}\text { Modulus } \\
{[\mathrm{GPa}]}\end{array}$ \\
\hline This work & Py-LCGO/PAN/PDA & Wet spinning & $\mathbf{7 3 8}$ & $\mathbf{7 3 . 2}$ \\
{$[17]$} & PAN grafted GO & Wet spinning & 452 & 8.3 \\
{$[36]$} & Glycidyl methacrylate grafted GO & Wet spinning & 500 & \\
{$[31]$} & Hyperbranched polyglycerol GO & Wet spinning & 652 & 20.9 \\
{$[32]$} & Cellulose/GO & Wet spinning & 442 & 34.1 \\
{$[37]$} & Ag-doped GO & Wet spinning & 300 & 9.5 \\
{$[38]$} & Reduced GO & Wet spinning & 360 & 12.8 \\
{$[9]$} & $\mathrm{GO}$ coagulated by CaCl & Wet spinning & 412 & 20.1 \\
{$[39]$} & Reduced GO/Poly (vinyl alcohol) & Wet spinning & 162 & 10.4 \\
{$[2]$} & Reduced GO/CNT/Poly(vinyl alcohol) & Wet spinning & 570 & \\
{$[33]$} & $800^{\circ} \mathrm{C}$ thermally treated GO & Wet spinning & 420 & \\
{$[34]$} & $1000{ }^{\circ} \mathrm{C}$ thermally treated GO & Wet spinning & 214 & 47 \\
{$[35]$} & $1050^{\circ} \mathrm{C}$ thermally treated GO & Wet spinning & 378 & 36.2 \\
{$[28]$} & $1800{ }^{\circ} \mathrm{C}$ thermally treated GO & Wet spinning & 1080 & \\
{$[40]$} & $\mathrm{GO}$ fiber from film & Twisting & 85 & 1.6 \\
{$[41]$} & $\mathrm{GO}$ coagulated by CaCl & & 136 & 7.9 \\
\hline
\end{tabular}

